\title{
TILLING in forage grasses for gene discovery and breeding improvement
}

Chloe Manzanares ${ }^{\S}$, Steven Yates ${ }^{\S}$, Michael Ruckle, Michelle Nay and *Bruno Studer

Forage Crop Genetics, Institute of Agricultural Sciences, ETH Zurich, 8092 Zurich, Switzerland

${ }^{\S}$ These authors contributed equally to this work

*Corresponding author: bruno.studer@usys.ethz.ch

Keywords: Breeding, Forage grass, Forward genetics, Perennial ryegrass (Lolium perenne L.), Reverse Genetics, Targeting Induced Local Lesions IN Genomes (TILLING) 


\begin{abstract}
Mutation breeding has a long-standing history and in some major crop species, many of the most important cultivars have their origin in germplasm generated by mutation induction. For almost two decades, methods for TILLING (Targeting Induced Local Lesions IN Genomes) have been established in model plant species such as Arabidopsis (Arabidopsis thaliana L.), enabling the functional analysis of genes. Recent advances in mutation detection by second generation sequencing technology have brought its utility to major crop species. However, it has remained difficult to apply similar approaches in forage and turf grasses, mainly due to their outbreeding nature maintained by an efficient self-incompatibility system.

Starting with a description of the extent to which traditional mutagenesis methods have contributed to crop yield increase in the past, this review focuses on technological approaches to implement TILLING-based strategies for the improvement of forage grass breeding through forward and reverse genetics. We present first results from TILLING in allogamous forage grasses for traits such as stress tolerance and evaluate prospects for rapid implementation of beneficial alleles to forage grass breeding.

In conclusion, large-scale induced mutation resources, used for forward genetic screens, constitute a valuable tool to increase the genetic diversity for breeding and can be generated with relatively small investments in forage grasses. Furthermore, large libraries of sequenced mutations can be readily established, providing enhanced opportunities to discover mutations in genes controlling traits of agricultural importance and to study gene functions by reverse genetics.
\end{abstract}




\section{Introduction}

\section{Agronomical challenges caused by changing socio-economic and climatic environments}

World food security is of increasing public concern [1]. The growing demand for food, feed and fuel, increasing scarcity of water and arable land, climate change and rising energy prices requires an increase in plant productivity, but in an ecologically sustainable way [2,3]. The development of crops with high and stable yields of good quality is a cornerstone of sustainable, intensified agricultural production. In order to realize these traits in future crops, novel tools that combine a broad range of technologies and innovative breeding strategies are needed [4].

\section{Plant breeding - an effective tool to increase productivity}

Plant breeding is the process to genetically improve crops for desired traits by selection. It mainly consists of the generation of variability in the breeding nursery, selection and extensive testing of the selected material to either be developed into a new variety or to be introduced into the next breeding cycle (Figure 1, black). Over the past 50 years, plant breeding has attained a nearly linear increase in the yield of many major crop species such as maize (Zea mays L.), rice (Oriza sativa L.) and wheat (Triticum aestivum L.). However, the high relative rates of yield increase during the green revolution have since been steadily declining. Moreover, yield increases are below those expected to meet the predicted demands by $2050[5]$.

Grasslands are important ecosystems; worldwide, they account for $80 \%$ of milk production and $70 \%$ of meat production [6]. Perennial ryegrass (Lolium perenne L.) is a major component of temperate grassland systems. In forage grasses, biomass is the primary yield target, but despite intensive breeding efforts over the last decades, increases in biomass yield are below that of major crop species [7]. Due to a highly effective self-incompatibility system [8], forage grasses are mainly improved as population and synthetic varieties which is considered as one of the main reasons for the limited annual breeding progress. Moreover, self-incompatibility, by hampering self-pollination, maintains heterozygosity and recessive mutations are not directly exhibited in the phenotype. 


\section{Biotechnological opportunities to advance breeding}

The rapid development of modern breeding methods, not solely based on transgenic modification, and the increasing understanding of crop genetics and genomics offer new possibilities to enhance breeding efficiency and precisely target the genes controlling desirable traits [9]. Approaches to advance breeding can be categorized in four areas: i) technology to unlock and increase the genetic diversity that is available for breeding, ii) approaches to accelerate the breeding cycle, iii) methods to increase the selection efficiency and iv) emerging bioinformatics tools to assist plant breeding (Figure 1, coloured in dark red, blue, green and red, respectively). In this review, we will focus on the first area and discuss how beneficial alleles can be identified, generated and used in a breeding program. In particular, we evaluate the potential of TILLING in allogamous forage grasses, as an efficient method to induce beneficial mutations and go beyond the natural genetic diversity to advance breeding for sustainable intensification.

\section{Exploitation of the natural genetic variation for breeding}

Genetically diverse material forms the foundation of every breeding program and for centuries, breeding advances have arisen through exploitation and recombination of the natural variation available in the gene pool of a species. Natural genetic diversity is a valuable and rich source to improve traits such as environmental resilience [10]. Through evolution, plants have adapted to virtually every terrestrial ecosystem, including extremes such as deserts, high altitude and salty marshes. Historically, Darwin described adaption as a consequence of natural selection [11]. For adaption to happen, new traits need to occur on which natural selection can act on. De Vries discovered such 'jump like' changes in pure-bred stocks and called them mutations [12], even before the discovery of DNA [13]. Schrödinger inferred that in order to enable adaption, mutations need to occur at low frequencies, otherwise more common harmful mutations would dominate over the few advantageous mutations in an organism [14]. Although plant breeders have selected spontaneous mutations in crops as early as $300 \mathrm{BC}$, it was not until after the realization of Mendel's laws of inheritance that mutants were actively screened and targeted by breeding to increase diversity and specificity of traits available for crop improvement.

To date, allelic variation is classically introduced from wild relatives, landrace varieties and previously undomesticated species [15]. However, the introgression of beneficial alleles from 
exotic germplasm may be limited by epistatic interactions, which decrease their efficacy [16]. Even if the allele does confer a beneficial quantitative effect on the phenotype, the crossing of exotic germplasm (donor) into elite material (recipient) can lead to the introgression of deleterious alleles reducing performance. As a consequence, recurrent selection is required to purge the deleterious alleles and to restore the elite material to its original performance [17]. Such a process can be greatly accelerated by use of marker assisted selection, but the introgression of wild alleles into modern breeding germplasm remains laborious and challenging [18].

\section{Targeted induction of genetic variation by genome editing}

In order to overcome the limitations of classical introgression breeding, modern methods offer opportunities to target specific genes of interest and directly introduce allelic variability by genome editing technologies [19]. Beside genome editing via zinc-finger nucleases (ZFNs) [20] and transcription activator-like effector nucleases (TALENs) [21], the clustered regularly interspaced short palindromic repeat (CRISPR)/Cas systems (e.g., Cas9) has become the preferred genome editing tool, largely due to its efficiency, specificity and flexibility [22,23]. While the basic proof of concept of the CRISPR/Cas9 technology is being established in many organisms, researchers are already beginning to apply this gene editing technology for crop trait improvement [24].

However, for the successful transition of the CRISPR/Cas9 system from the lab into the field, some major crop-specific challenges remain, for example, the availability of efficient transformation protocols, promoters and vectors [22]. Moreover, the genotypes used to implement the CRISPR/Cas9 system in the lab are often selected for high responsiveness to transformation and tissue culture, being genetically dissimilar to advanced breeding germplasm. This poses the same difficulties as described above for the introgression of novel alleles from exotic germplasm. Also, genome editing technologies are currently creating indistinct boundaries in the regulatory framework of genetically modified organisms and create new challenges for the regulation and social acceptance of these technologies [24]. 


\section{Modern mutation induction to increase and exploit genetic diversity for breeding}

In contrast to targeted gene modification, random mutations can also affect the function of a gene, albeit in a less specific manner. Mutations in plants can occur spontaneously, for example through DNA replication errors, or can be induced at much higher frequencies by exposing plants or seeds to radiation, UV-light, chemical treatments or by T-DNA insertional mutagenesis [25]. Radiation induces deletions in the genome, which can range in size from large (mega base pairs) to small (single base pairs), while chemical mutagens typically alter single base pairs [26]. The most frequently used radiation mutagenesis sources are gamma and X-rays [27], while N-nitroso-N-methyl urea (NMU), ethyl methanesulfonate (EMS) or sodium azide $\left(\mathrm{NaN}_{3}\right)$ are commonly used chemical mutagens [28]. Mutation induction to increase the genetic diversity for breeding offers a number of attractive benefits. First, the mutations can be created in contemporary germplasm, and although the mutagenesis affects the entire genome and may induce undesired epistatic effects, the number of selection cycles used to remove the mutations causing these effects and to establish the desired phenotype in advanced breeding germplasm is significantly reduced compared to introgression of exotic germplasm. Second, the quantitative effect of the mutation is active in the relevant genetic background. This circumvents the difficulties in the introgression of quantitative trait loci (QTL) from exotic germplasm, where beneficial alleles from one population (QTL donor) might not be effective in alternative genetic backgrounds (QTL recipient) due to epistasis [16$18]$.

Traditional mutation breeding has a long-standing history and was attributable to over 3,200 mutant varieties that have been released [29]. Various ancestors of today's crop varieties originated from mutation breeding [30]. For example, several barley (Hordeum vulgare L.) varieties used in the brewing industry today are progenies of the high-yielding, short heighted, mutation-bred 'Diamant' and 'Golden Promise' cultivars [30,31]. In durum wheat (Triticum durum L.), the semi dwarf 'Cappelli' mutant 'Cp B144', created through thermal neutron irradiation, was used for the development of the widely adopted variety 'Creso' in Italy [32]. When released in 1974, 'Creso' increased durum wheat production by $0.9 \mathrm{t} / \mathrm{ha}$ resulting in an increased revenue of USD\$ 180 million per year [30]. Together, the two examples above, along with other semi dwarf varieties, have added billions of USD dollars in value [30]. Rice has also benefited from mutation breeding, for example the cold tolerant 'Kashmir Basmati' 
cultivar in Pakistan. This variety combined early flowering with high grain quality and cold tolerance, which allowed this cultivar to be grown at higher altitudes [33]. Other examples of mutation breeding lead to the improvement of cold, salinity and biotic stress tolerance as well as semi-dwarf, lodging resistant cultivars with better grain quality [34] with over 500 cultivars incorporating mutagenized material [35].

\section{Forward genetics: from trait to gene}

Forward genetics aims at determining the genetic basis responsible for a phenotype. The starting point is an observed phenotype, which is then genetically dissected to identify the underlying mutation. In the context of mutation breeding, the phenotypes are the product of mutagenesis. Key to any forward genetics approach is an efficient phenotyping method, whereby desirable phenotypes can be readily distinguished from the wild-type [36]. The choice of the mutagenizing agent influences the means available to identify the causal mutation [37]. The use of biological agents, for example T-DNA insertion or retrotransposons, has the advantage that the inserted DNA can be used as a template to find the position, although such methods are species-specific and not applicable to all plants [38]. In contrast, mutations induced through chemical or radiation treatments are applicable in any species. However, without novel sequence introgression, map-based cloning approaches are necessary to locate the causal mutation [39].

A forward genetic screen typically begins with mutagenesis of seed material. Seeds are the easiest target for mutagenesis, as they can be planted directly after the treatment to obtain the $\mathrm{M}_{1}$ generation. Additionally, due to their small size, large quantities of material can be subject to the mutagenesis treatment. The use of a genetically homogeneous starting population $\left(\mathrm{M}_{0}\right)$ is desirable for forward genetic screens as divergent phenotypes are likely the product of mutagenesis, as opposed to phenotypic differences which may be the result from a heterologous genetic composition of the $\mathrm{M}_{0}$ population. The germination and growth of mutagenized seed can be informative of the effect of the mutagenesis treatment, where the mutation frequency can be estimated by measuring the growth, or more accurately by the frequency of albino plants in the $M_{1}$ [29]. Mutagenesis rates can differ by orders of magnitude between species, but in mutable species, one mutation per $100-200 \mathrm{~kb}$ is possible [40]. The frequency of successfully identifying a mutant for a specific phenotype depends on several factors, including the number of genes controlling it. If the mutant screen is specific to a desired phenotype governed by a small number of genes, the frequency of obtaining a mutant 
can be low and mutagenesis of tens of thousands of seeds are generally needed [38]. One major limitation to these screens is that the $\mathrm{M}_{1}$ generation typically contains heterozygous mutations and therefore, only dominant mutations will directly be exhibited in the phenotype. This limitation is avoided in self-pollinating plants such as Arabidopsis, which can be propagated to the $\mathrm{M}_{2}$ generation, where in diploids, one fourth of the offspring are homozygous for the induced mutation. In out-crossing plants, where self-pollination is not possible, methods to concentrate the mutagenized alleles such as the "crossing within spike progeny" have been described [41].

From a desirable phenotype, identified through forward genetic screens, the location of the causal mutation can be found by a number of methods. Classically, this is done by map-based cloning, which typically involves crossing the mutant plant with a wild type plant to produce a mapping population. Molecular markers associated with the mutation will co-segregate in the offspring with the mutant phenotype, and by analysis of recombination events, the approximate location of the causal mutation can be determined [42]. Depending on the size of the mapping population, these regions often contain several genes and might need further dissection [43]. Today, the tools for positional cloning have evolved substantially with the use of next-generation sequencing (NGS). In contrast to genotyping individual members of a segregating population, sequencing pools of individuals with contrasting phenotypes can be more effective. In so called bulk segregant analyses, contrasting pools typically containing hundreds of individuals are sequenced by NGS to identify the causal mutation based on allele frequency differences between the pools [44]. These techniques do not require a complete reference genome and therefore are not limited to model organisms as reviewed by Schneeberger [45].

\section{Forward genetic screens in model plant and major crop species}

The success of forward genetic screens combined with technological advances in functional genomics has led to the extensive understanding of the genes that control biological systems in plants. The vast majority of this knowledge was derived from studies using the model plant Arabidopsis. From a review of the Arabidopsis Gene Ontology evidence codes (downloaded from http://plants.ensembl.org/, 07/09/2015), the function of 9,185 genes are inferred from direct assays (IDA), 842 are inferred from gene interaction (IGI) and 4,032 are inferred from mutant phenotypes (IMP). Thus, of the 27,417 genes in Arabidopsis, approximately one third have experimental evidence (the sum of the IDA, IGI and IMP equals to 10,266) to support 
their function. In contrast, only a small percentage of genes have the same level of experimental evidence to substantiate their function in rice (IDA, 223; IGI, 9 and IMP, 130). In forage grasses, there might be a similarly large repertoire of genes whose function can be inferred by forward genetic screens. Although promising for genes controlling mechanisms that are conserved throughout angiosperms [46], evidence of gene function may not directly translate from Arabidopsis into grasses [47], due to the evolutionary distance between monocots and dicots diverging 130 to 150 Mya [48,49].

Technical opportunities, enabled by NGS, to identify the causal mutation of a phenotype are particularly useful in combination with previously established large-scale populations of phenotyped plants. For example in rice, the International Rice Research Institute has generated 60,000 IR64 mutants of which 38,000 lines have been taken to M4 generation [50]. The Swedish mutation research program has so far catalogued over 12,000 barley mutants, with a range of morphological mutations [51]. Moreover, forward genetic screens have been used to study traits and mechanisms including osmotic stress [52,53], heat stress [54], phytohormone activities [55-57], seed development [58], fatty acid synthesis [59] and biotic stress [60].

\section{Forward genetic screens in forage grasses}

Although forward genetic screens have been widely used in many model and major crop species, it is an underutilized tool in forage grass breeding. With a view to assess the potential of forward genetic screens to increase abiotic stress tolerance in perennial ryegrass, we have screened 100,000 chemically mutagenized $\mathrm{M}_{1}$ seeds for tolerance to salt $(\mathrm{NaCl})$, water stress (induced by polyethylene glycol, PEG) and the stress-signalling phytohormone abscisic acid (ABA) during germination (Figure 2A). Forty nine putative salt tolerant mutants (Figure 2B), 22 putative osmotic stress tolerant mutants and 16 putative ABA insensitive mutants were identified. Tolerant phenotypes were distinguished from normal phenotypes by germinating or enhanced root growth on media containing the stressor. The identified putative mutants were grown, clonally multiplied and will undergo extensive field testing to evaluate if the increased abiotic stress tolerance identified at the seedling stage translate into the field.

To demonstrate the effectiveness of this forward genetic screen, the perennial ryegrass homolog of ETHYLENE OVERPRODUCER1 (LPETO1) was further investigated. ETO1 was identified from a mutant screen of around $20,000 \mathrm{M}_{2}$ Arabidopsis seedlings that were 
subjected to saline stress and a dominant loss of function allele was identified as the cause of soil-salinity tolerance [61]. In the 49 putative salt tolerant mutants of perennial ryegrass, the LPETO1 gene was sequenced. A total of five potential mutations were found, two of which were non-synonymous. Given these preliminary data, the mutation frequency was estimated to approximately one per $11.7 \mathrm{~kb}$ in LPETO1. The high mutation rate observed in LPETO1 is indicative of an enrichment of functional mutations through the forward screening and provides preliminary evidence that LPETO1 effects salt tolerance in perennial ryegrass. However, the evaluation of the mutation rate in neighbouring and housekeeping genes is required to fully conclude that the forward screening has enriched the mutation rate in this particular gene.

\section{Reverse genetics: from gene to trait}

Reverse genetics describes the process to discover the function of a gene by evaluating the phenotypic effect of an induced gene disruption. Opposite to forward genetics, it starts with the genotype or predicted gene and evaluates the phenotype. Reverse genetics is becoming increasingly popular as the availability of NGS continually expands, as well as the establishment of techniques that can be readily applied to a broad range of species regardless of established genetic resources. Arguably, the most adaptable reverse genetics method is TILLING. TILLING identifies individuals in a population that contain allelic variation in a target gene. Such allelic variants can be induced by the same mutagenesis treatments as described for forward genetics, or through the use of a wild or diverse genetic population, which has been termed ecoTILLING [62]. Alleles found by TILLING approaches are valuable for functional genomic studies, particularly in species where genetic modification is not possible due to limited transformation protocols. Moreover, TILLING is a promising tool to transfer knowledge gained from model systems such as Arabidopsis to crop plants and since TILLING does not fall under GMO regulations [63], research material can directly be translated to the field for crop improvement.

\section{TILLING in model plant and major crop species}

The first reported use of TILLING was in Arabidopsis, where a TILLING population was developed to determine the biological function of several enzymes by identifying plants that contained mutations in the coding sequence of the annotated genes [64]. Since then, TILLING has been widely adapted to identify mutants with specific phenotypes. When applied to crop 
species, these mutations can translate into specific traits that are predicted from the reported gene function. A number of companies and institutions have established TILLING platforms for a number of crops such as wheat [63], maize [65], pea (Pisum sativum L.) [66], tomato (Solanum lycopersicum L.) [67], amongst others. One of the largest public TILLING resource centers is the URGV - Plant Genomic Research in Versailles (France), where 14 TILLING collections in eight crop species have been developed, which feed into 30 research projects (http://www-urgv.versailles.inra.fr/tilling/projects.htm).

Successful examples of developed traits in crop species have been reported. In tomato, over 3,000 EMS mutant lines were used to identify beneficial alleles of fruit ripening related genes, and TILLED mutants remained intact 60 days after harvesting [68]. In cucumber (Cucumis sativus L.), over 3,000 EMS $\mathrm{M}_{2}$ lines were created to identify mutants with favourable sexual traits [69]. In wheat, two EMS-mutagenized populations were created in hexaploid and tetraploid varieties, where 246 alleles for granule bound starch synthase (GBS/WAXY) were found. Selected alleles were used to produce low amylose content starch [63]. In barley, an EMS derived population of over $10,000 \mathrm{M}_{2}$ plants was screened for a homeodomain-leucinezipper protein (HD-ZIP) of which several mutants exhibited altered spikelet morphology [70].

\section{TILLING in forage grasses}

With the recent release of the draft genome sequence of perennial ryegrass [71], TILLING in forage grasses becomes more attractive. The identification of relevant homologs of interesting target genes from model species in perennial ryegrass is significantly more practical. Moreover, the recent advancements in NGS and its adaptation to TILLING have significantly increased the throughput and the number of candidate genes that can be screened by TILLING [72].

In order to exploit the potential of TILLING as a functional genomics platform in forage grasses, three different populations of perennial ryegrass containing 550, 1350 and $1700 \mathrm{M}_{1}$ plants, respectively, were established. Each population represents an $F_{1}$ cross progeny and contains a maximum of four alleles before the mutagenesis, which minimizes the allelic diversity of the population background. The mutagenesis was done by treating the seeds with $\mathrm{NaN}_{3}$. Preliminary tests with different $\mathrm{NaN}_{3}$ concentrations showed that each population responded significantly differently to $\mathrm{NaN}_{3}$, based on the germination rates determined for each population. Therefore, the treatment intensity needed to be optimised for each 
population. Finally, two of the populations were treated with $1 \mathrm{mM}$ of $\mathrm{NaN}_{3}$, resulting in $30 \%$ and $77 \%$ germination rate, respectively. The third population was treated with a higher concentration of $1.5 \mathrm{mM}$ of $\mathrm{NaN}_{3}$, resulting in a $61 \%$ germination rate.

The germinated seeds of the three populations were grown and transplanted in separated fields as individual plants. Due to self-incompatibility in perennial ryegrass, it was not possible to create, like in other species, homozygotes $\mathbf{M}_{2}$ plants derived from self-pollination. This challenge was resolved by applying a modified strategy as described in Ukai [41], where $\mathrm{M}_{2}$ seeds were obtained by polycrosses separated for each population. The $\mathrm{M}_{2}$ seeds were harvested separately for each individual plant. With this method, interesting mutations will be heterozygous in the polycross progeny $\left(\mathrm{M}_{2}\right)$. In the case of an interesting mutation, the $\mathrm{M}_{2}$ seeds will be germinated, offspring containing the mutation identified by an efficient genotyping approach [73] and subjected to self-pollination. Though laborious, it is possible to force self-pollination in perennial ryegrass [74]. Although this will result in a limited number of seeds, it is the most efficient method to obtain homozygous $\mathrm{M}_{3}$ plants.

In order to identify mutations, a sequencing-based approach was used. A tissue sample from a single inflorescence was collected for DNA extraction and sequencing. The seeds from the same inflorescence were harvested and are considered as the $\mathrm{M}_{2}$ seeds. This strategy was used in order to reduce the percentage of chimerism. Chimerism is a common phenomenon in random mutation induction as the induced mutations affect individual cells in the embryo differently. As a consequence, cells inducing inflorescences and gametes will not always carry the same mutations in the $\mathrm{M}_{1}$, leading to the segregation of different mutations in the next generation. Therefore, chimerism is a problem when working with $\mathrm{M}_{1}$ plants. By sampling leaf material directly emerging from the inflorescence, the mutation identified in the leaf is more likely to be present in the $\mathrm{M}_{2}$ seeds. Current sequencing-based methods for mutation detection make use of two or three dimensional pooling to reduce library preparation costs for sequencing [75]. However, in a heterozygous population with a range of haplotypes, such pooling would give rise to unique profiles of polymorphisms within a pool. We anticipate this may limit the detection of mutations. Furthermore, short read sequencing poses challenges in reconstituting haplotypes [76]. To overcome these problems, we envision an amplicon-based sequencing strategy; instead of high dimensional pooling, a high scalable barcoding strategy will be used to barcode each genotype individually. Amplicons will be sequenced using the Pac-Bio SMRT technology where circular consensus sequencing will 
enable high accuracy haplotyping of genotypes [77]. This strategy will allow efficient identification of induced mutations by comparing haplotypes within the TILLING population.

\section{Potential and challenges of TILLING in forage grasses}

\section{Challenges of TILLING in forage grasses}

Although promising, several unknowns and specific challenges remain for the application of TILLING to the improvement of perennial ryegrass. The first major challenge is the limited knowledge on the mutability. Feasible TILLING platforms require mutation frequencies within the range of one mutation per $100 \mathrm{~kb}-500 \mathrm{~kb}$. These frequencies are estimated by the allelic variation needed to achieve a desired phenotype, the size of the target gene and practical limitations on mutagenized population size. Lower mutation frequencies, smaller genes and the need for greater allelic variation (more than 20 mutations are generally needed to identify a useful allele) can greatly increase the size of the required population [78].

Another challenge in many forage grasses is the generation time. Indeed, unlike Arabidopsis, which has a short generation time of around 7 to 8 weeks [79], perennial ryegrass requires vernalization and therefore around one year to complete a generation cycle. In a TILLING population, plants need to go through several generations in order to have recessive mutations in the homozygous state or to remove any other mutations that could interfere with the phenotype. An additional challenge is the self-incompatibility mechanism of many important forage grasses, hampering efficient self-pollination and thus complicating and further delaying the process to exhibit recessive mutations in the phenotype. These two specificities are inevitably increasing the time needed to obtain a TILLING population and introduce beneficial alleles into breeding programs. The use of self-compatible germplasm $[80,81]$ as a basis for the development of TILLING populations can facilitate and speed up the implementation of TILLING by at least one generation.

Because TILLING-based reverse genetics requires the transfer of knowledge from model species to forage grasses, it is difficult to assess the relevance this knowledge will have on an expected trait. In the majority of successful TILLING examples, the target trait is typically monogenic. In forage grasses, the primary trait is biomass yield, which is multigenic and generally defined by epistatic trade-offs. For example, heading date, which has been described to be influenced by allelic variation in $L p H D 1$, the perennial ryegrass homolog of the Arabidopsis gene CONSTANS, also impacts biomass, persistency and forage quality [82]. 
However, few tools exist to aid in the selection of candidate genes from model species, as resources such as the Gene Ontology are not readily applicable for trait prediction.

\section{Potential of TILLING for functional genomics and breeding applications in forage grasses}

At the early stages, our results demonstrate the potential of combining forward and reverse genetics concepts in perennial ryegrass for gene discovery and breeding improvement. The putative salt and osmotic stress tolerant mutants, identified through forward genetic screens, have an applicable trait in contemporary breeding programs. Moreover, putative ABA insensitive mutants are useful for the elucidation of stress signalling pathways to understand how stress response is coupled to plant growth and yield formation, for example under water limited conditions [83].

Perennial ryegrass as a forage crop is not typically planted and grown in saline environments, in part because it does not exhibit salt tolerance [84]. Because salt tolerance mechanisms share a common regulatory network with other osmotic stresses, salt insensitive mutants have also been found to have improved tolerance to other stresses [85-87]. When perennial ryegrass is bred and used as turf, salinity tolerance is an important trait, because water used for irrigation can contain high salt concentrations that limit turf grass growth and establishment $[88,89]$. Whilst salt tolerance has already improved by genetic modification [89], finding novel mechanisms involved in germination and growth under saline conditions through forward genetics could directly translate into turf grass breeding.

\section{Conclusions}

For decades, mutation induction has been used to increase the genetic diversity in many crop species and produced useful alleles, which have contributed to the significant yield increases during the green revolution. In forage grasses, mutagenized seeds, in combination with innovative screening strategies, can be used to identify beneficial alleles for important traits such as abiotic stress resilience. Here, we present an example of the potential of such forward genetic screens by the identification of putative ABA insensitive, salt and osmotic stress tolerant mutants in contemporary breeding germplasm. Initial sequencing of the LPETO1 gene in putative salt tolerant mutants resulted in the discovery of novel mutations in this gene. ETO1 has been shown in Arabidopsis to have a quantitative effect on salt resistance [61]. Therefore, we hypothesize that the high mutation rate observed in LpETO1 is not representative of the mutation frequency across the genome, but due to the selection for 
modulation of the LPETO1 gene function with the described forward genetic screen. Given the successful identification of novel LPETO1 alleles, we assert that the mutagenesis treatment described here, when applied to TILLING, will result in further allele discovery in target genes.

The full potential of TILLING as a classical reverse genetics tool in forage grasses has yet to be exploited. One reason why TILLING has found limited application is the presence of selfincompatibility in major forage grasses, hampering self-pollination and thus the ability to exhibit recessive mutations directly in the phenotype. The availability of self-fertile plant material will be a major step forward to realize efficient TILLING approaches. Moreover, continuous technical advancements in NGS and the recently published draft of the perennial ryegrass genome sequence will further facilitate efficient identification of induced mutations by pooled DNA sequencing. Ultimately, TILLING strategies will need to further evolve to take full advantage of the continuing advancements in sequencing technologies. For example, the use of single molecule sequencing to TILLING-based mutation screens has the potential to identify haplotypes from chimeric plants. Moreover, we anticipate that highly scalable barcoding schemes may replace multi-dimensional pooling which will provide an effective means to identify mutations in heterozygous populations. The TILLING strategy outlined here can also be applied in other outbreeding crop species and will help to unlock the potential of modern mutation induction methods for crop improvement in forage grasses and other orphan crops.

\section{Acknowledgements}

The authors would like to acknowledge Martina Birrer and Pietro Delfino for excellent technical support, Deutsche Saatveredelung AG for providing the seeds used for TILLING as well as Mario Kurmann and the Forage Crop Genetics group for help with the plant material. We sincerely thank Prof. Dr. Achim Walter for hosting the Forage Crop Genetics group at ETH Zurich.

This work was supported by the Swiss National Science Foundation (SNSF Professorship grant no: PP00P2 138988) and the European Union's Seventh Framework Programme for research, technological development and demonstration under grant agreement no: GA-2010267243 - PLANT FELLOWS. 


\section{References}

[1]. Godfray HC, Beddington JR, Crute IR, Haddad L, Lawrence D, Muir JF, et al. Food security: the challenge of feeding 9 billion people. Science, 2010; 327: 812-818.

[2]. Cominelli E, Conti L, Tonelli C, Galbiati M. Challenges and perspectives to improve crop drought and salinity tolerance. New Biotechnol, 2013; 30: 355-361.

[3]. Wheeler T, von Braun J. Climate Change Impacts on Global Food Security. Science, 2013; 341: 508-513.

[4]. Tester M, Langridge P. Breeding technologies to increase crop production in a changing world. Science, 2010; 327: 818-822.

[5]. Fischer T, Byerlee D, Edmeades G. Crop yields and global food security: Will yield increase continue to feed the world? Canberra, Australia: Australian Center for International Agricultural Research; 2014.

[6]. Wilkins PW, Humphreys MO. Progress in breeding perennial forage grasses for temperate agriculture. J Agr Sci, 2003; 140: 129-150.

[7]. van der Heijden SAG, Roulund N. Genetic gain in agronomic value of forage crops and turf: A review. Sustainable Use of Genetic Diversity in Forage and Turf Breeding 2010; 247-260.

[8]. Cornish MA, Hayward MD, Lawrence MJ. Self-incompatibility in ryegrass. I. Genetic control in diploid Lolium perenne L. Heredity, 1979; 43: 95-106.

[9]. Kopecký D, Studer B. Emerging technologies advancing forage and turf grass genomics. Biotech Adv, 2014; 32: 190-199

[10]. Tanksley SD, Mccouch SR. Seed Banks and Molecular Maps: Unlocking Genetic Potential From the Wild. Science, 1997; 277: 1063-1066. 
[11]. Darwin. The origin of species by means of natural selection, or the preservation of favored races in the struggle for life. John Murray, London 1859.

[12]. De Vries H. The mutation theory: Open Court Publishing Company; 1909.

[13]. Watson JD, Crick FH. Molecular structure of nucleic acids. Nature, 1953; 171: 737-738.

[14]. Schrödinger E (1944) What is life? The physical aspect of the living cell. Cambridge, NY. The University Press.

[15]. Hajjar R, Hodgkin T. The use of wild relatives in crop improvement: A survey of developments over the last 20 years. Euphytica, 2007; 156: 1-13.

[16]. Collins NC, Tardieu F, Tuberosa R. Quantitative trait loci and crop performance under abiotic stress: Where do we stand? Plant Physiol, 2008; 147: 469-486.

[17]. Podlich DW, Winkler CR, Cooper M. Mapping as you go: An effective approach for marker-assisted selection of complex traits. Crop Sci, 2004; 44: 1560-1571.

[18]. Kumar A, Dixit S, Ram T, Yadaw RB, Mishra KK, Mandal NP. Breeding high-yielding drought-tolerant rice: genetic variations and conventional and molecular approaches. $\mathbf{J}$ Exp Bot, 2014; 65: 6265-6278.

[19]. Gaj T, Gersbach CA, Barbas CF. ZFN, TALEN, and CRISPR/Cas-based methods for genome engineering. Trends Biotech, 2013; 31: 397-405.

[20]. Townsend JA, Wright DA, Winfrey RJ, Fu FL, Maeder ML, Joung JK, et al. Highfrequency modification of plant genes using engineered zinc-finger nucleases. Nature, 2009; 459: 442-445.

[21]. Bogdanove AJ, Voytas DF. TAL effectors: Customizable proteins for DNA targeting. Science, 2011; 333: 1843-1846. 
[22]. Schaeffer SM, Nakata PA. CRISPR/Cas9-mediated genome editing and gene replacement in plants: Transitioning from lab to field. Plant Sci, 2015; 240: 130-142.

[23]. Belhaj K, Chaparro-Garcia A, Kamoun S, Patron NJ, Nekrasov V. Editing plant genomes with CRISPR/Cas9. Current Opinion in Biotech, 2015; 32: 76-84.

[24]. Araki M, Ishii T. Towards social acceptance of plant breeding by genome editing. Trends Plant Sci, 2015; 20: 145-149.

[25]. Alonso JM, Stepanova AN, Leisse TJ, Kim CJ, Chen H, Shinn P, et al. Genome-wide insertional mutagenesis of Arabidopsis thaliana. Science, 2003; 301: 653-657.

[26]. Shu Q, Shirasawa K, Hoffmann M, Hurlebaus J, Nishio T, Forster B, et al. Molecular techniques and methods for mutation detection and screening in plants. In: Shu Q, Forster BP, Nakagawa H. Plant mutation breeding and biotechnology 2012; p.241256.

[27]. Mba C, Afza R, Shu Q, Forster B, Nakagawa H. Mutagenic radiations: X-rays, ionizing particles and ultraviolet. In: Shu Q, Forster BP, Nakagawa H. Plant mutation breeding and biotechnology 2012; p.83-90.

[28]. Leitao J, Shu Q, Forster B, Nakagawa H. Chemical mutagenesis. In: Shu Q, Forster BP, Nakagawa H. Plant mutation breeding and biotechnology 2012; p.135-158.

[29]. FAO (2014) Statistical Yearbook 2014: World Food and Agriculture. Rome, Italy: FAO (Food and Agriculture Organziation of the United Nations).

[30]. Ahloowalia B, Maluszynski M, Nichterlein K. Global impact of mutation-derived varieties. Euphytica, 2004; 135: 187-204.

[31]. Forster BP. Mutation genetics of salt tolerance in barley: An assessment of Golden Promise and other semi-dwarf mutants. Euphytica, 2001; 120: 317-328. 
[32]. Maluszynski M, Szarejko I. Induced mutations in the Green and Gene Revolutions. In: Tuberosa R, Phillips RL, Gale M, editors; Bologna, Italy. Avenue media, Bologna. 2003; p. 403-425.

[33]. Bashir K, Khan NM, Rasheed S, Salim M. Indica rice varietal development in Pakistan: an overview. Paddy Water Environ, 2007; 5: 73-81.

[34]. Maluszynski M, Ahloowalia B, Ashri A, Nichterlein K, Van Zanten L. Induced mutations in rice breeding and germplasm enhancement. In: Assessment and orientation towards the 21 st century 1998; 194-204.

[35]. Tai TH. Induced mutations in rice (Oryza sativa L.). Isr J Plant Sci, 2007; 55: 137-145.

[36]. Page DR, Grossniklaus U. The art and design of genetic screens: Arabidopsis thaliana. Nat Rev Genet, 2002; 3: 124-136.

[37]. Peters JL, Cnudde F, Gerats T. Forward genetics and map-based cloning approaches. Trends Plant Sci, 2003; 8: 484-491.

[38]. Krysan PJ, Young JC, Sussman MR. T-DNA as an insertional mutagen in Arabidopsis. Plant Cell 1999; 11: 2283-2290.

[39]. Lukowitz W, Gillmor CS, Scheible WR. Positional cloning in Arabidopsis. Why it feels good to have a genome initiative working for you. Plant Physiol, 2000; 123: 795-805.

[40]. Tsai H, Missirian V, Ngo KJ, Tran RK, Chan SR, Sundaresan V, et al. Production of a High-Efficiency TILLING Population through Polyploidization. Plant Physiol, 2013; 161: 1604-1614.

[41]. Ukai Y. "Crossing-Within-Spike-Progeny method"; An effective method for selection of mutants in cross-fertilizing plants. Technical News of the Institute of Radiation Breeding 1983; 25. 
[42]. Peleman JD, Van Der Voort JR. Breeding by Design. Trends Plant Sci, 2003; 8: 330334.

[43]. Mackay TF, Stone EA, Ayroles JF. The genetics of quantitative traits: challenges and prospects. Nat Rev Genet, 2009; 10: 565-577.

[44]. Schneeberger K, Ossowski S, Lanz C, Juul T, Petersen AH, Nielsen KL, et al. SHOREmap: simultaneous mapping and mutation identification by deep sequencing. Nat Methods, 2009; 6: 550-551.

[45]. Schneeberger K. Using next-generation sequencing to isolate mutant genes from forward genetic screens. Nat Rev Genet, 2014; 15: 662-676.

[46]. Blumel M, Dally N, Jung C. Flowering time regulation in crops - what did we learn from Arabidopsis? Curr Opin Biotechnol, 2015; 32: 121-129.

[47]. Spannagl M, Mayer K, Durner J, Haberer G, Frohlich A. Exploring the genomes: from Arabidopsis to crops. J Plant Physiol, 2011; 168: 3-8.

[48]. Chaw SM, Chang CC, Chen HL, Li WH. Dating the monocot-dicot divergence and the origin of core eudicots using whole chloroplast genomes. J Mol Evol, 2004; 58: 424441.

[49]. Hertweck KL, Kinney MS, Stuart SA, Maurin O, Mathews S, Chase MW, et al. Phylogenetics, divergence times and diversification from three genomic partitions in monocots. Bot J Linn Soc, 2015; 178: 375-393.

[50]. Wu JL, Wu CJ, Lei CL, Baraoidan M, Bordeos A, Madamba MRS, et al. Chemical- and irradiation-induced mutants of indica rice IR64 for forward and reverse genetics. Plant Mol Biol, 2005; 59: 85-97.

[51]. Lundqvist U. Scandinavian mutation research in barley - a historical review. Hereditas, 2014; 151: 123-131. 
[52]. Koiwa H, Bressan RA, Hasegawa PM. Identification of plant stress-responsive determinants in Arabidopsis by large-scale forward genetic screens. J Exp Bot, 2006; 57: 1119-1128.

[53]. Vallejo AJ, Yanovsky MJ, Botto JF. Germination variation in Arabidopsis thaliana accessions under moderate osmotic and salt stresses. Ann Bot, 2010; 106: 833-842.

[54]. Guan QM, Yue XL, Zeng HT, Zhu JH. The protein phosphatase RCF2 and its interacting partner NAC019 are critical for heat stress-responsive gene regulation and thermotolerance in Arabidopsis. Plant Cell, 2014; 26: 438-453.

[55]. Bleecker AB, Estelle MA, Somerville C, Kende H. Insensitivity to ethylene conferred by a dominant mutation in Arabidopsis thaliana. Science, 1988; 241: 1086-1089.

[56]. Koornneef M, Reuling G, Karssen CM. The isolation and characterization of abscisicacid insensitive mutants of Arabidopsis thaliana. Physiol Plantarum, 1984; 61: $377-$ 383.

[57]. Maher EP, Martindale SJB. Mutants of Arabidopsis thaliana with altered responses to auxins and gravity. Bioch Genet, 1980; 18: 1041-1053.

[58]. McElver J, Tzafrir I, Aux G, Rogers R, Ashby C, Smith K, et al. Insertional mutagenesis of genes required for seed development in Arabidopsis thaliana. Genetics, 2001; 159: 1751-1763.

[59]. Lemieux B, Miquel M, Somerville C, Browse J. Mutants of Arabidopsis with alterations in seed lipid fatty-acid composition. Theor Appl Genet, 1990; 80: 234-240.

[60]. Krothapalli K, Buescher EM, Li X, Brown E, Chapple C, Dilkes BP, et al. Forward genetics by genome sequencing reveals that rapid cyanide release deters insect herbivory of Sorghum bicolor. Genetics, 2013; 195: 309-318. 
[61]. Jiang C, Belfield EJ, Cao Y, Smith JAC, Harberd NP. An Arabidopsis soil-salinitytolerance mutation confers ethylene-mediated enhancement of sodium/potassium homeostasis. Plant Cell, 2013; 25: 3535-3552.

[62]. Comai L, Young K, Till BJ, Reynolds SH, Greene EA, Codomo CA, et al. Efficient discovery of DNA polymorphisms in natural populations by Ecotilling. Plant J, 2004; 37: 778-786.

[63]. Slade A, Fuerstenberg S, Loeffler D, Steine M, Facciotti D. A reverse genetic, nontransgenic approach to wheat crop improvement by TILLING. Nat Biotech, 2005; 23: $75-81$.

[64]. McCallum CM, Comai L, Greene EA, Henikoff S. Targeting Induced Local Lesions IN Genomes (TILLING) for plant functional genomics. Plant Physiol, 2000; 123: 439442.

[65]. Till B, Reynolds S, Weil C, Springer N, Burtner C, Young K, et al. Discovery of induced point mutations in maize genes by TILLING. BMC Plant Biol, 2004; 4: 12.

[66]. Dalmais M, Schmidt J, Le Signor C, Moussy F, Burstin J, Savois V, et al. UTILLdb, a Pisum sativum in silico forward and reverse genetics tool. Genome Biol, 2008; 9: R43.

[67]. Piron F, Nicolaï M, Minoïa S, Piednoir E, Moretti A, Salgues A, et al. An Induced Mutation in Tomato eIF4E Leads to Immunity to Two Potyviruses. PLoS ONE, 2010; 5: e11313.

[68]. Okabe Y, Asamizu E, Saito T, Matsukura C, Ariizumi T, Brès C, et al. Tomato TILLING technology: development of a reverse genetics tool for the efficient isolation of mutants from Micro-Tom mutant libraries. Plant Cell Physiol, 2011; 52: 1994-2005.

[69]. Boualem A, Fleurier S, Troadec C, Audigier P, Kumar AP, Chatterjee M, et al. Development of a Cucumis sativus TILLinG Platform for Forward and Reverse Genetics. PLoS ONE, 2014; 5: e97963. 
[70]. Gottwald S, Bauer P, Komatsuda T, Lundqvist U, Stein N. TILLING in the two-rowed barley cultivar 'Barke' reveals preferred sites of functional diversity in the gene HvHox1. BMC Res Notes, 2009; 2: 258.

[71]. Byrne SL, Nagy I, Pfeifer M, Armstead I, Swain S, Studer B, et al. A synteny-based draft genome sequence of the forage grass Lolium perenne. Plant J, 2015; 84: 816-826.

[72]. Tsai H, Howell T, Nitcher R, Missirian V, Watson B, Ngo KJ, et al. Discovery of rare mutations in populations: TILLING by sequencing. Plant Physiol, 2011; 156: 12571268.

[73]. Studer B, Jensen LB, Fiil A, Asp T. "Blind" mapping of genic DNA sequence polymorphisms in Lolium perenne L. by high resolution melting curve analysis. Mol Breed, 2009; 24: 191-199.

[74]. Wilkins PW, Thorogood D. Breakdown of self-incompatibility in perennial ryegrass at high temperature and its uses in breeding. Euphytica, 1992; 64: 65-69.

[75]. Henry IM, Nagalakshmi U, Lieberman MC, Ngo KJ, Krasileva KV, Vasquez-Gross H, et al. Efficient genome-wide detection and cataloging of EMS-induced mutations using exome capture and next-generation sequencing. Plant Cell 2014; 26: 1382-1397.

[76]. Snyder MW, Adey A, Kitzman JO, Shendure J. Haplotype-resolved genome sequencing: experimental methods and applications. Nat Rev Genet, 2015; 16: 344-358.

[77]. Travers KJ, Chin CS, Rank DR, Eid JS, Turner SW. A flexible and efficient template format for circular consensus sequencing and SNP detection. Nucleic Acids Res, $2010 ; 38$.

[78]. Hädrich N, Gibon Y, Schudoma C, Altmann T, Lunn JE, Stitt M. Use of TILLING and robotised enzyme assays to generate an allelic series of Arabidopsis thaliana mutants with altered ADP-glucose pyrophosphorylase activity. J Plant Physiol, 2011; 168: 1395-1405. 
[79]. Baud S, Boutin J, Miquel M, Lepiniec L, Rochat C. An integrated overview of seed development in Arabidopsis thaliana ecotype WS. Plant Physiol Bioch, 2002; 40: 151160.

[80]. Thorogood D, Kaiser WJ, Jones JG, Armstead I. Self-incompatibility in ryegrass 12. Genotyping and mapping the $S$ and $Z$ loci of Lolium perenne L. Heredity, 2002; 88: 385-390.

[81]. Thorogood D, Armstead IP, Turner LB, Humphreys MO, Hayward MD. Identification and mode of action of self-compatibility loci in Lolium perenne L. Heredity, 2005; 94: 356-363.

[82]. Skot L, Humphreys J, Humphreys MO, Thorogood D, Gallagher J, Sanderson R, et al. Association of candidate genes with flowering time and water-soluble carbohydrate content in Lolium perenne (L.). Genetics, 2007; 177: 535-547.

[83]. Claeys H, Inzé D. The agony of choice: how plants balance growth and survival under water-limiting conditions. Plant Physiol, 2013; 162: 1768-1779.

[84]. Venables AV, Wilkins D. Salt tolerance in pasture grasses. New Phytol, 1978; 80: 613622.

[85]. Werner JE, Finkelstein RR. Arabidopsis mutants with reduced response to $\mathrm{NaCl}$ and osmotic stress. Physiol Plantarum, 1995; 93: 659-666.

[86]. Quesada V, Ponce MR, Micol JL. Genetic analysis of salt-tolerant mutants in Arabidopsis thaliana. Genetics, 2000; 154: 421-436.

[87]. Wu L, Zhang Z, Zhang H, Wang X-C, Huang R. Transcriptional modulation of ethylene response factor protein JERF3 in the oxidative stress response enhances tolerance of tobacco seedlings to salt, drought, and freezing. Plant physiol, 2008; 148: 1953-1963.

[88]. Brilman L. Salt Tolerance of Perennial Ryegrass Varieties. Seed research of Oregon 2008 
[89]. Wu YY, Chen QJ, Chen M, Chen J, Wang XC. Salt-tolerant transgenic perennial ryegrass (Lolium perenne L.) obtained by Agrobacterium tumefaciens-mediated transformation of the vacuolar $\mathrm{Na}^{+} / \mathrm{H}^{+}$antiporter gene. Plant Sci, 2005; 169: 65-73. 


\section{Figure legends}

Figure 1: Simplified breeding cycle (black) and technological approaches (coloured) to advance plant breeding. The methods are categorized into i) technology to unlock and increase the genetic diversity (including exploitation of natural genetic resources, genome editing and TILLING; dark red), ii) approaches to accelerate the breeding cycle (including speed breeding and tissue culture methods such as double haploid induction; blue), iii) methods to increase the selection efficiency (including automated phenotyping, markerassisted and genomic selection; green) and iv) emerging bioinformatics tools to assist plant breeding (red).

Figure 2: Osmotic stress and forward screen stressors. A shows the stress perception and the downstream response in plants. Three abiotic stressors (salt, cold and drought) are shown which limit water uptake. In response to osmotic stress, plants produce the phytohormone abscisic acid (ABA), which initiates a stress response through transcription factors (TF) and $\mathrm{Ca}^{2+}$ ions, resulting in reactive oxygen species (ROS) scavenger production, osmotic adjustment, cell wall hardening and stomatal closure. B shows the results from a forward screen where mutagenized seeds were germinated on $75 \mu \mathrm{M}$ ABA, the magnified red box shows a single seed germinating on ABA stressor media.

Figure 3: TILLING as a reverse genetics tool in perennial ryegrass (Lolium perenne L.). A Outline of the TILLING strategy applied to perennial ryegrass in order to identify and characterise mutants. $\mathbf{B} \mathbf{M}_{1}$ plants resulting from mutagenized seeds were germinated and transplanted into the field. $\mathbf{M}_{2}$ seeds derived from polycrossed $\mathbf{M}_{1}$ plants were harvested, along with the flag leaf of the inflorescence in order to minimize chimerism $(\mathbf{C}) . \mathbf{M}_{2}$ seeds from identified $M_{1}$ mutants will be germinated and screened for the mutation. $M_{2}$ plants containing the mutation will be self-pollinated in order to obtain $\mathrm{M}_{3}$ seeds. $\mathrm{M}_{3}$ plants homozygous for the mutation will then be phenotyped in order to characterise the mutation. 


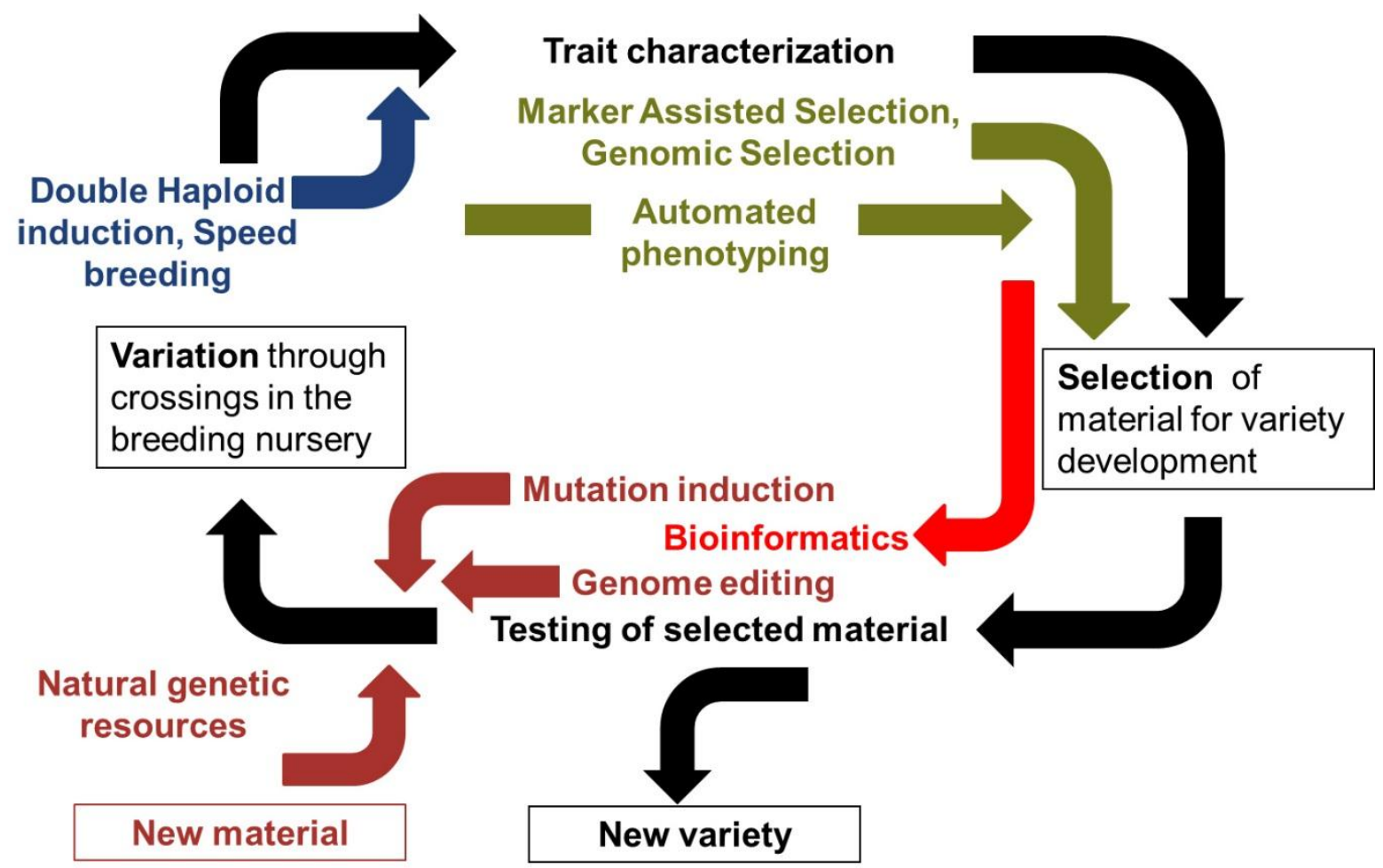




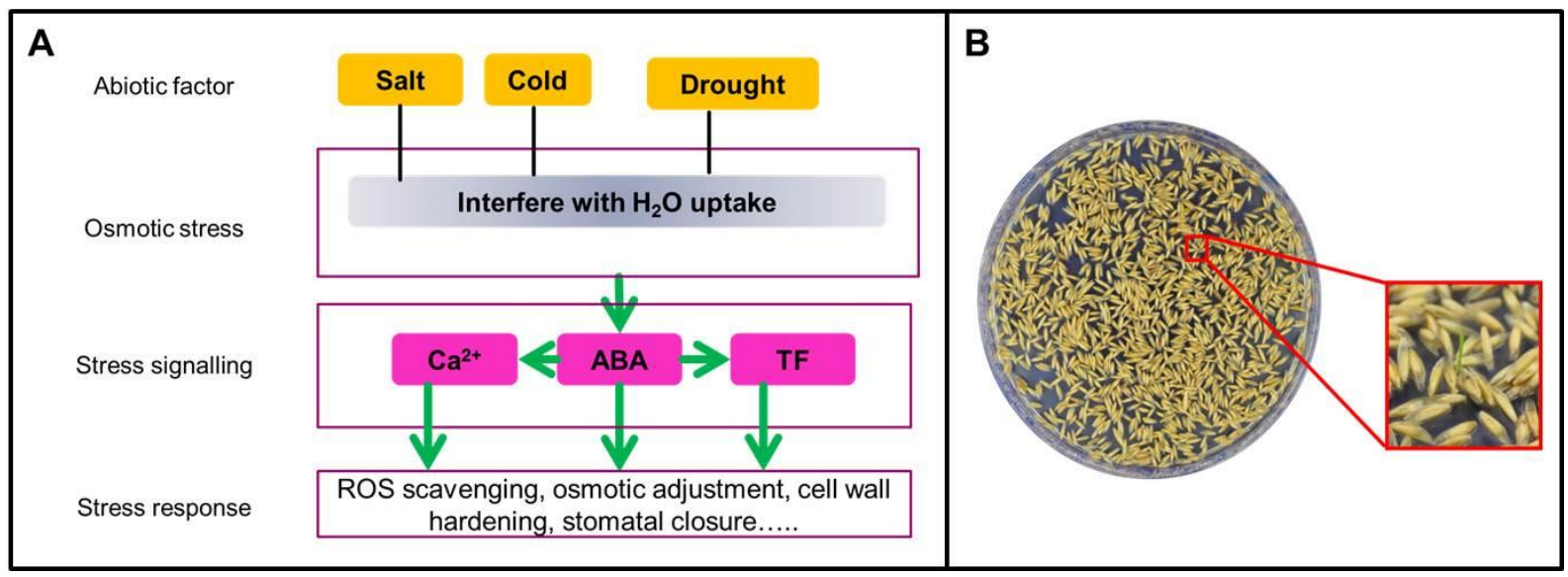




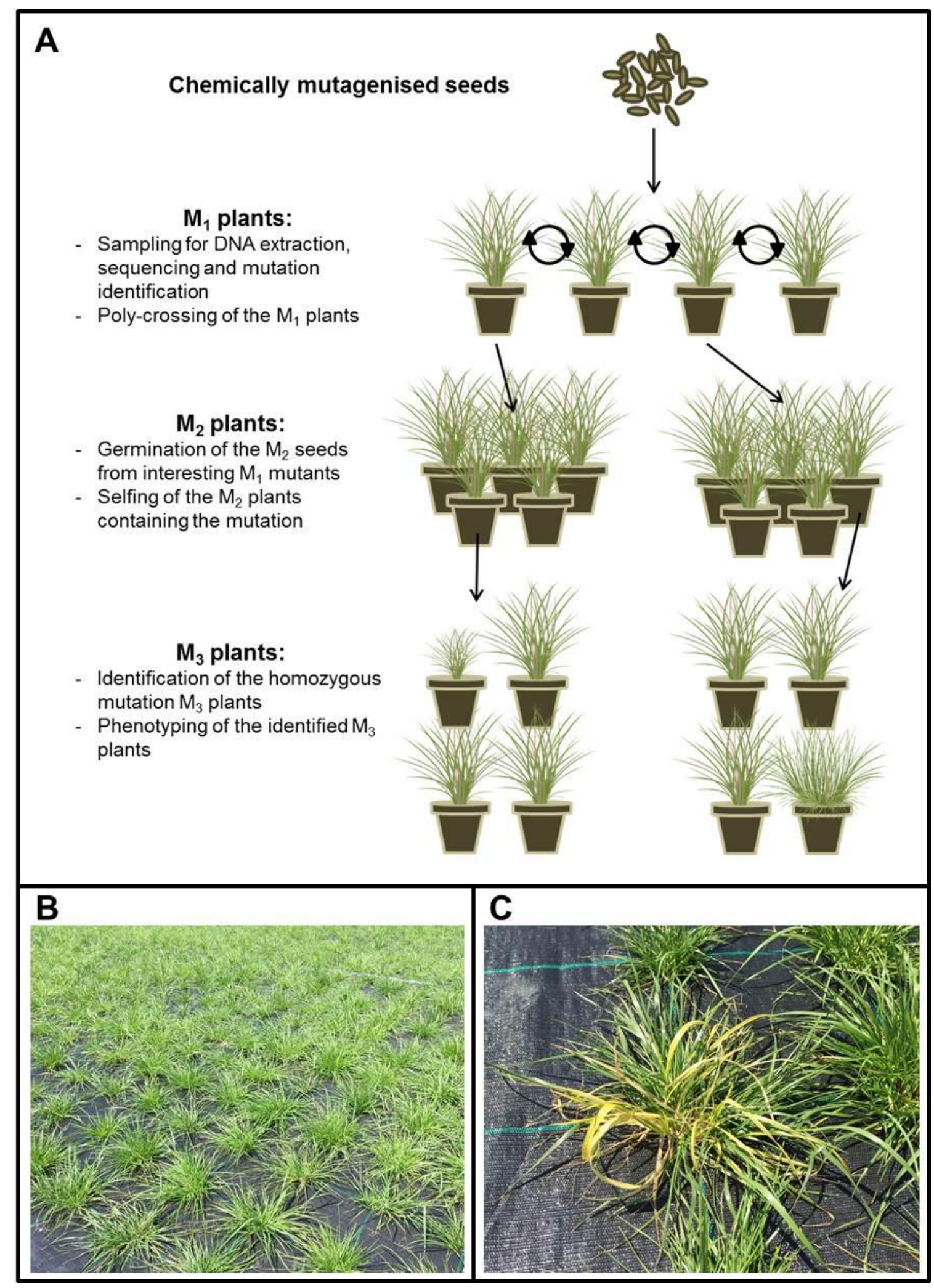

